\title{
Evolving Role of Three-Dimensional Echocardiography in the Cardiac Surgical Patient
}

\author{
Renata G. Ferreira $\cdot$ Yong Seon Choi • \\ G. Burkhard Mackensen
}

Published online: 30 July 2013

(C) Springer Science + Business Media New York 2013

\begin{abstract}
The first human three-dimensional transesophageal echocardiography (3D TEE) was reported in 1992. Ever since, technologic advances have revolutionized this echocardiographic imaging modality. Today, real-time 3D TEE (RT-3D TEE) represents a novel clinical and intuitively educational perioperative cardiovascular imaging tool. In addition, live 3D TEE adds significantly to the perioperative communication between cardiac surgeons and echocardiographer. Its unique ability of real-time acquisition and accurate identification of precise cardiac pathology, together with its ability to promptly quantify 3D datasets using builtin software, has greatly helped to transition 3D TEE into the standard of perioperative care. The recent development of percutaneous procedures such as valve implantations and repairs also benefit from 3D TEE quantification and guidance. This article reviews the available 3D TEE acquisition
\end{abstract}

Electronic supplementary material The online version of this article (doi:10.1007/s40140-013-0025-6) contains supplementary material, which is available to authorized users.

R. G. Ferreira · G. B. Mackensen ( $\square)$

Division of Cardiothoracic Anesthesiology, Department of Anesthesiology \& Pain Medicine, University of Washington, 1959 N.E. Pacific Street, Room BB1461, Seattle, WA 98195, USA

e-mail: gbmac@uw.edu

R. G. Ferreira

e-mail: rf50@uw.edu

Y. S. Choi

Department of Anesthesiology and Pain Medicine, Yonsei University College of Medicine, Seoul, Republic of Korea e-mail: ychoi12@uw.edu

Y. S. Choi

1959 N.E. Pacific Street, Room BB1461, Seattle, WA 98195 , USA modes, newest advances in 3D technology, and the utility of 3D TEE in different settings of cardiac procedures.

Keywords Cardiac surgery - Transesophageal three-dimensional echocardiography .

Perioperative assessment

\section{Introduction}

In the last decade, one of the most important developments in ultrasound imaging of the heart has been the evolution of $3 \mathrm{D}$ echocardiography from slow and labor-intense offline reconstruction to real-time volumetric imaging [1].

In light of these recent and ongoing improvements of 3D echocardiography, echocardiographers are constantly expanding its use in quantifying valvular heart disease, ventricular function, or when assessing complex cardiac anatomy. Intraoperatively, the introduction of RT-3D TEE has quickly been embraced by both cardiac surgeons and anesthesiologists, as this new technology offers superior image quality, greater accuracy, and ever-expanding capabilities. The interventional cardiology suite and the hybrid operating room are other areas where the advent of RT-3D TEE has revolutionized procedural imaging and guidance as well as the immediate diagnosis of related complications.

Significant interest in 3D technology has been sparked in the research community due to its growing availability, its ease of use, and its multiple attractive features, resulting in a large number of related publications and guidelines within the last decade $[2,3 \cdot \bullet, 4 \cdot]$ The purpose of this article is to review current $3 \mathrm{D}$ imaging technologies and the utility of $3 \mathrm{D}$ echocardiography in the perioperative period. Each intraoperative use of 3D echocardiography will be described individually along with its technical limitations. 


\section{D Imaging Modes and Post-Processing}

3D images can currently be acquired in two different ways: 3D live or 3D ECG gated imaging. 3D live or RT-3D TEE refers to the acquisition of the image by utilizing multiple pyramidal datasets per second in a single heartbeat. In 3D ECG gated imaging, data acquisition is triggered by the ECG and multiple beats are used to build the final image. There are currently two notable ultrasound systems built for 3D/4D image processing, iE33 xMatrix (Philips Medical Systems, Andover, MA, USA) and Vivid E 9 (GE Healthcare Vingmed Ultrasound, Horten, Norway). The available modes of 3D/4D acquisition are described in more detail below:

\section{Real Time Three-Dimensional Echocardiography (3D Live)}

This mode displays in real time $300 \times 600$ pyramidal volumes. It can be used to conveniently display cardiac structures located in the near field (Fig. 1). This mode results in superior spatial and temporal resolution permitting accurate diagnoses of complex pathologies. In general, RT-3D TEE or live 3D is preferentially used to display the mitral valve (MV), aortic valve (AV), the left atrial appendage (LAA) and the intraatrial septum.

\section{Focused Sector-Zoom}

Also known as 3D Zoom (Philips Medical Systems) or FlexiZoom (GE Healthcare Vingmed Ultrasound), this mode displays a magnified pyramidal dataset of variable size. It permits a focused visualization of a specific cardiac structure or region. It is important to note that, in this 3D acquisition mode, the focused area of interest being imaged is obtained at the cost of the decrease in spatial and temporal resolution. Careful placement and sizing of the zoom sector over the region of interest with minimized sector-width improves temporal resolution and optimizes image quality (Fig. 2). Live 3D color zoom is a new feature available on the most updated version of iE33, Vision 2012 (Philips Medical Systems). In the operative room, this new tool can potentially improve the accuracy in the assessment of postoperative valvular regurgitation. In the interventional laboratory, it helps to assess structural heart defects as well as to diagnose paravalvular leaks after valve deployment in transcatheter aortic valve implantation (TAVI) procedures.

\section{Full Volume}

This mode provides the largest pyramidal dataset possible (approximately $65^{\circ} \times 60^{\circ}$ up to $100^{\circ} \times 100^{\circ}$ ). When utilizing the conventional 3D workflow, the wide-angle dataset is compiled by either acquiring data based on a single heart beat or by merging two to six narrower RT-3D pyramidal wedges or subvolumes obtained over two to six heartbeats. Available in the most update version of the iE33, Vision 2012, HVR mode consists of a method of scanning a live $3 \mathrm{D}$ volume using subvolumes. Traditionally, a subvolume consists of a contiguous subset of scan lines. An advantage of using contiguous subsets is that there are few locations where the different subsets join and therefore a small number of locations where "stitching" artifacts would be visible. A disadvantage of using contiguous subsets is that, when a given subset is being scanned, there is no information about the remainder of the volume. As such, when there is significant motion of either the patient or the transducer, it is necessary to completely rescan the entire volume to acquire an acceptable full volume. With HVR mode, the volume is scanned with subvolumes that consist of interlocking sparse patterns. An example of a classic interlocking sparse pattern is a chessboard (Fig. 3). This figure shows the difference between the traditional acquisition of the subvolumes and the HVR mode lateral and axial elevation views. An advantage of using interlocking sparse patterns is that, while one pattern is being scanned, it is possible to make a good estimate of the complete volume by interpolating the missing samples. A disadvantage relates to the potential for many "stitching" artifacts given the many locations where the different patterns join. In the HVR mode, scanning alternates between subvolumes. During one cardiac cycle, one subvolume is repeatedly scanned. During the next cardiac cycle, other subvolumes are repeatedly scanned. While one subvolume is being scanned, the missing subvolume is estimated using the surrounding subvolumes.

New software has been developed to help improve the acquisition of large volumes of 3D data. The one beat acquisition available at the Vision 2012 and the 4D views are both "one-touch" options to quickly acquire images of larger structures such as left ventricle.

For the conventional 3D full volume acquisition it is important to minimize artifacts. During the surgical or interventional procedure in the anesthetized patient, full volume loops should be acquired when ventilation is held and electrocautery is not used. In patients with arrhythmia artifacts cannot be totally avoided. After optimization of the two-dimensional (2D) image of the four-chamber view, a full volume loop of the left ventricle (LV) is acquired. Once the full volume mode is activated, a biplane image with the four-chamber view and the correspondent orthogonal plane is displayed on the screen. The first obtained 3D-volume is displayed as auto crop showing only $50 \%$ of the volume mirroring the original fourchamber view. Resetting the crop plane allows the display 
Fig. 1 RT-3D TEE en face (or surgical) view of the MV demonstrating the aortic valve at 12 o'clock in the near field. $A V$ aortic valve, $M V$ mitral valve

Fig. 2 3D TEE focused sector images of the MV. 3D Zoom feature (Philips; top) and FlexiZoom (GE Healthcare; bottom) $A V$ Aortic valve, $P L$ posterior leaflet
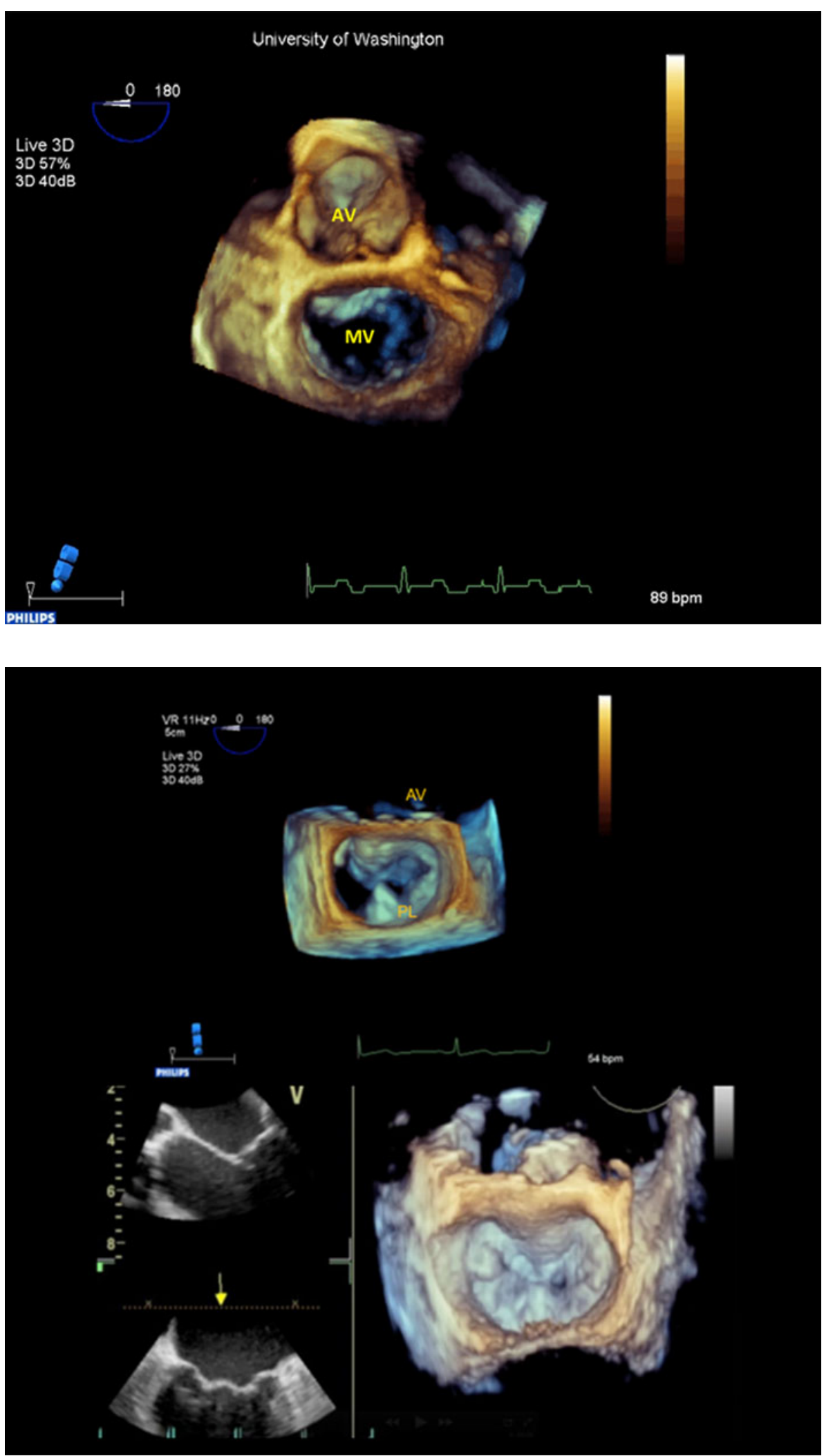

of the entire pyramidal dataset. The full volume can be further processed offline by rotating and cropping to visualize specific structures inside the pyramid. Acquired full volumes can also be used for volumetric quantification of the LV using the available built-in software features explained below. 


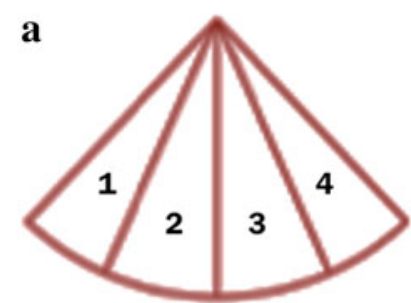

Lateral elevation view

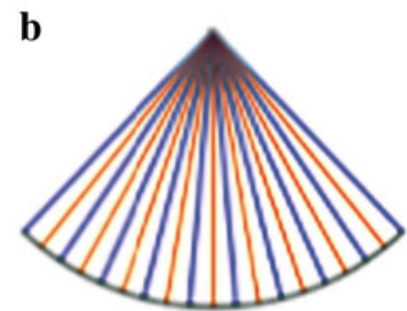

Lateral elevation view

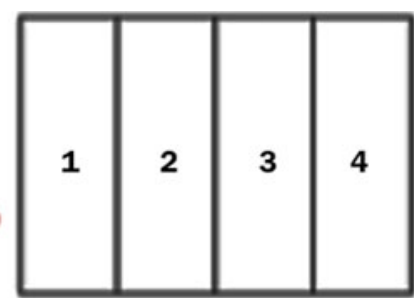

Axial elevation view

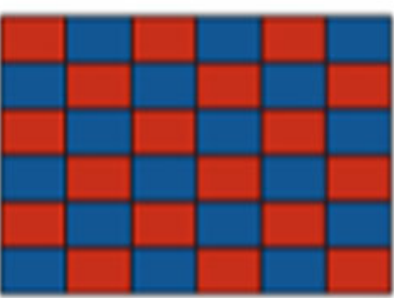

Axial elevation view

Fig. 3 HVR mode for full volume acquisition. a The contiguous subset of scan lines in axial and lateral elevation views. b HVR mode demonstrating how the interlocking space patterns look in the lateral elevation and in the axial view. Reproduced with permission from Philips Healthcare

\section{Full Volume with Color Flow Doppler}

The acquisition of the 3D full volume with color Doppler is very similar to the regular full volume data acquisition. It consists of the integration of 7-14 (original version of iE33) or 2-6 individual pyramidal subvolumes gated to the ECG.

Similarly to the full volume, this mode is prone to artifacts introduced by arrhythmias, movement, or electrocautery. For this mode, it is essential to place the area of interest, e.g., a regurgitant or patent foramen ovale (PFO) jet in the center of the sector (Fig. 4; Movie Clip 1). Most updated software versions of 3D echocardiography allow for the acquisition of full volume with color flow in as few as two heartbeats.

\section{Clinical Applications}

Assessment of the Left Ventricle

Perhaps one of the most valuable contributions of 3D echocardiography in the perioperative period may be LV quantification [5]. The 3D echocardiographic assessment of LV volumes and function provides accuracies that approach the accuracy of cardiac magnetic resonance imaging (cMRI) [6-14]. Furthermore, 3D echocardiography for the assessment of $\mathrm{LV}$ volumes and ejection fraction (EF) has been currently recommended over the use of $2 \mathrm{D}$ echocardiography, due to its accuracy and reproducible measurements $[13,14]$. With the advent of the new semiautomated volumetric analysis systems, the global and segmental analysis of the LV has become less troublesome and less time-consuming which makes its intraoperative use more realistic.

One of the most utilized approaches to LV assessment in $3 \mathrm{D}$ is the acquisition of a full volume dataset. The software available for the analysis of LV function consists of built-in
Fig. 4 Full volume with color flow Doppler TEE data. Notice the area of interest, $P F O$, in the center of the sector

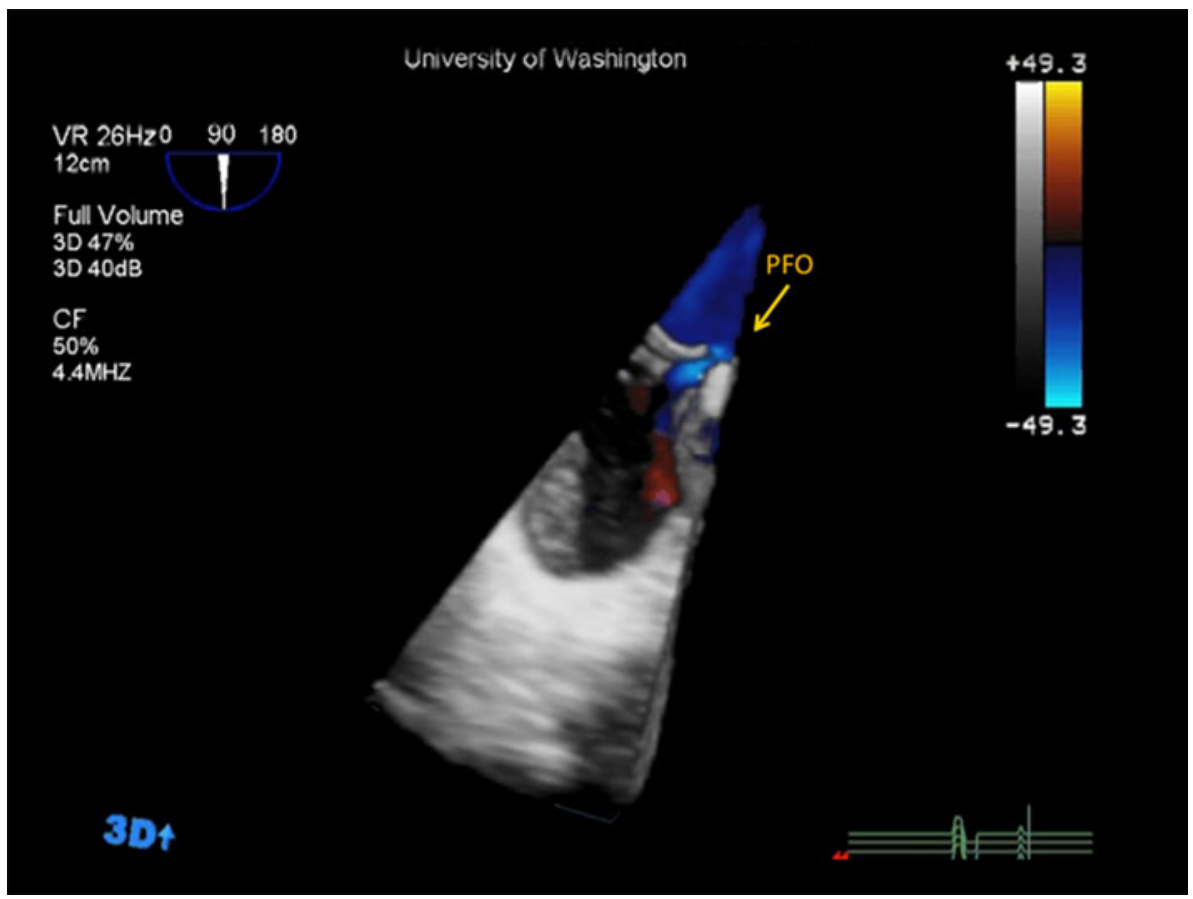


quantification features that are based on semiautomated endocardial border detection that allows fast and accurate measurements of global and regional LV function. Examples of available built-in software packages are 3D Quantification Advanced (3DQA) program (Philips Medical Systems) and 4D Auto left ventricular quantification LVQ (GE Healthcare Vingmed Ultrasound). Both vendors offer online as well as offline quantitative analysis.

The Philips 3DQA program consists of a mesh-based surface-tracking mode that provides automatic LV volume and EF. The system requires manual definition of the septal, lateral, anterior, inferior, and apical endocardial border of the LV in the end-systolic and the end-diastolic frames, followed by an automatic border-tracking algorithm. Further, the end-systolic as well as the end-diastolic volumes are calculated. Global stroke volume and EF are then derived. An advantage of this system is the segmental analysis of the left ventricle. The LV shell obtained in semiautomatic fashion is then subdivided in 17 regions, which are analyzed separately by performing the "segmental analysis". Other viewing modes include the "iSlice" view that displays nine simultaneously moving short axis views of the $\mathrm{LV}$ and allows verifying appropriate endocardial border detection as well as simultaneous wall motion abnormalities (Fig. 5).

An interesting advantage of the 4D is that, by utilizing temporal data, it delivers reproducible results that could be less prone to artifacts and variable heart rhythms for automatic volume and EF.

LV mass has previously been shown to be an independent predictor of incident cardiovascular disease morbidity and mortality [15]. Although rarely used during the perioperative period, this tool can be used to diagnose LV pathology such as hypertrophic cardiomyopathy [16]. The measurement of LV mass by 3D echocardiography correlated well with cMRI and demonstrated a lower interobserver variability than $2 \mathrm{D}$ echocardiography $[11,17-21]$. For the 4D LV mass, using the above-mentioned meshbased surface tracking model, by adding the epicardial border, an LV mass and an LV mass index can be derived from the dataset obtained for the LV EF.

To date, 2D transthoracic echocardiography remains the imaging modality of choice in assessment of LV clot. However increasing evidence in the literature shows that

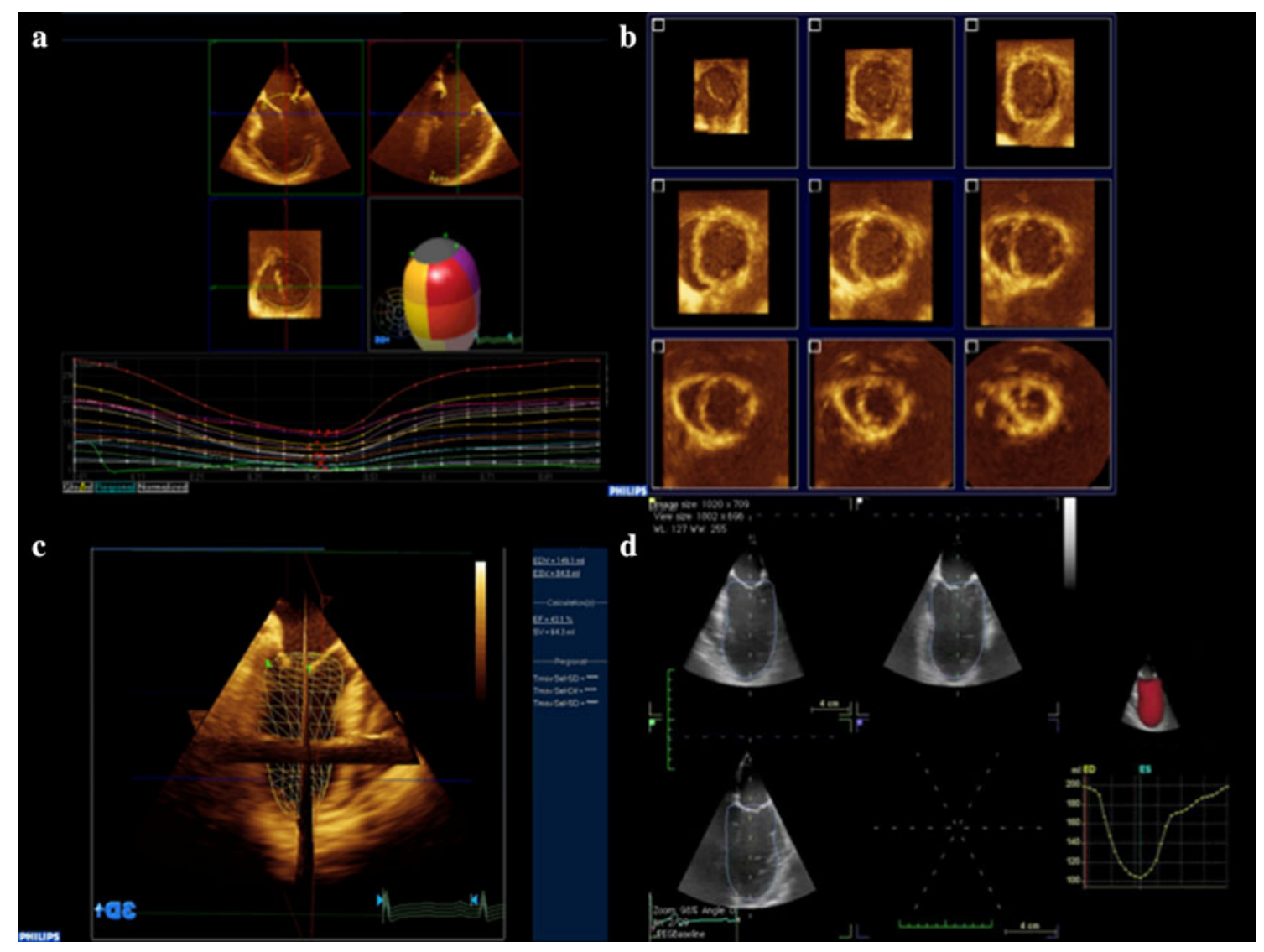

Fig. 5 3D datasets with different 3D visualization and analysis of the left ventricle (LV). a Segmental analysis of the LV with 17 segmental time-volume waveforms are displayed simultaneously offering the possibility for objective wall motion comparison, b "iSlice" 2D tomographic slicing of the LV. c "Mesh" of the LV d 4D Auto Left ventricular quantification (LVQ) (GEHEalthcare Vingmed Ultrasound) 
3D technology can be more accurate in intracardiac mass detection and should be considered in the diagnostic process [19].

\section{Assessment of the Right Ventricle}

Perioperative right ventricular (RV) dysfunction is one of the most frequent causes of morbidity and mortality in complex cardiac surgical patients. Nevertheless, global assessment of the $\mathrm{RV}$ is recognizably difficult mostly due to its geometric complexity. The 2D intraoperative assessment has several limitations, which can be overcome by 3D ECG gated image acquisition. Overall, 3D echocardiography allows the complete assessment of the RV anatomy, geometry, volumes, and EF [3••]. Recent work has shown that 3D TEE assessment of the RV and dedicated RV quantification software can be applied to reliably represent the complex geometry of the RV. Therefore, this approach constitutes a valuable tool for the assessment of the RV function during the perioperative period [22].

Recently, several methods and software packages have been developed to assess the RV. Similar to the LV, a 3D full volume dataset of the ME four chamber view is required. The stored data can be then processed offline. The 3D image of the RV is usually obtained from the ME fourchamber view with the probe slightly rotated to the right side of the patient in order to include the annulus of the RV.

A freestanding software (4D RV-Function; TomTec Imaging software, Unterschleissheim, Germany), dedicated to RV quantification facilitates the analysis of RV volume and function; this can be performed both with 3D TTE as well as with 3D TEE data [23]. After acquisition of the full-volume of the RV, TV and MV annulus, and the LV apex are manually marked (Fig. 6; Movie clips 2-3). These reference points are useful not only to define the tricuspid annulus but also to position the major right heart axis within the 3D dataset. The RV cavity is semiautomatically determined after the identification of the end- systolic and end-diastolic endocardial border. Measurements such as the end-systolic and end-diastolic volumes are automatically derived and the resulting stroke volume and EF calculated. The segmental analysis of the three main sections of the RV including inlet, apex, and outflow segments may be displayed [22].

Until the present time, gold standards methods for RV evaluation such as cMRI or radionucleotide ventriculography are not available intraoperatively. Although fasting and hemodynamic changes can make the evaluation of the RV function very challenging, 3D TEE might be the most feasible way to intraoperative evaluate the function of the right heart.
Mitral Valve Assessment

In the last decade, innumerous studies have been published in the literature comparing 2D versus 3D echocardiography in MV. [24-26] Most recently, Hien et al. demonstrated that 3D TEE is superior to 2D TEE for prolapsed and cord rupture [27]. The superiority of 3D echocardiography might be attributed to the non-planar 3D structure of the MV annulus; it is impossible to visualize it in a single plane using 2D TEE. Additionally, the saddle-shaped configuration of the MV and the complex interrelationships among the MV, chordae, papillary muscles, and myocardial walls mean that $3 \mathrm{D}$ imaging is particularly suited to achieve a complete assessment. Therefore, a comprehensive 2D examination requires interrogation of the MV apparatus at multiple planes and TEE probe positions and demands a mental integration of numerous views for accurate assessment which critically depends on observer experience and expertise. A great number of software packages have been developed to assist the intraoperative echocardiographer in the quantitative assessment of the MV [28, 29].

A comprehensive intraoperative evaluation of the MV involves the acquisition of the so-called en face view, a full volume and 3D color full volume image. Further analysis of the MV can be achieved by 3D quantitative assessment of the MV using built-in software (MVQ Qlaboratory, Philips Medical Systems; or MVA 2.0, TomTec Imaging software) (Fig. 7).

The en face or "surgical view" presents a view of the MV similar to that seen by the surgeon from a left atrial perspective. This view is routinely generated using the 3D zoom mode based on the ME four-chamber view and by rotating the obtained image to display the AV at the $12 \mathrm{o}$, clock position as the midpoint of the anterior annulus [30]., as previously shown in Fig. 1. The full volume dataset allows assessing the intimate interrelationship among the MV, the papillary muscles, the myocardial walls, and the left ventricular outflow tract (LVOT). 3D TEE has been used to identify the exact location of the pathology of mitral leaflet lesions in MV prolapsed (3 D4 and 5) (Movie Clip 4 and 5) should be here as opposed to (3D 4 and 5), endocarditis, and congenital MV abnormalities to guide the surgeon to optimize the surgical repair. According to a recent study, 3D TEE provided an objective tool for predicting MV repair complexity involving bileaflet repair techniques, requiring multiple resections or patch augmentation, in patients with mitral regurgitation (MR), in which the most predictive models included three predictors: multisegment pathology, prolapsing height, and posterior leaflet angle [31]. RT-3D TEE (94\% accuracy) was superior to RT-2D TEE (71\% accuracy) in accurately identifying abnormal MV segments in patients undergoing MV surgery for MR when compared to the surgical finding as a 
Fig. 6 Examples of the threedimensional evaluation of the RV based on a full volume dataset showing a RV with mild dysfunction. a Dynamic analysis of the RV with enddiastolic volume, end-systolic volume, and EF. b Threedimensional reconstruction can be visualized and rotated to assess from the wall side or from the ventricular side (b) move $\mathbf{b}$ to the beginning of the phrase consider remove the parentheses

Fig 7 Three-dimensional quantitative assessment MV using different software. 3D morphologic analysis of a normal MV demonstrating the commissural diameter (TomTec Imaging software) (top left). 3D animation of the MV annulus reconstructed from a real time 3D image of the MV also demonstrating the anterolateral to posterior medial diameter (Philips) (bottom right)
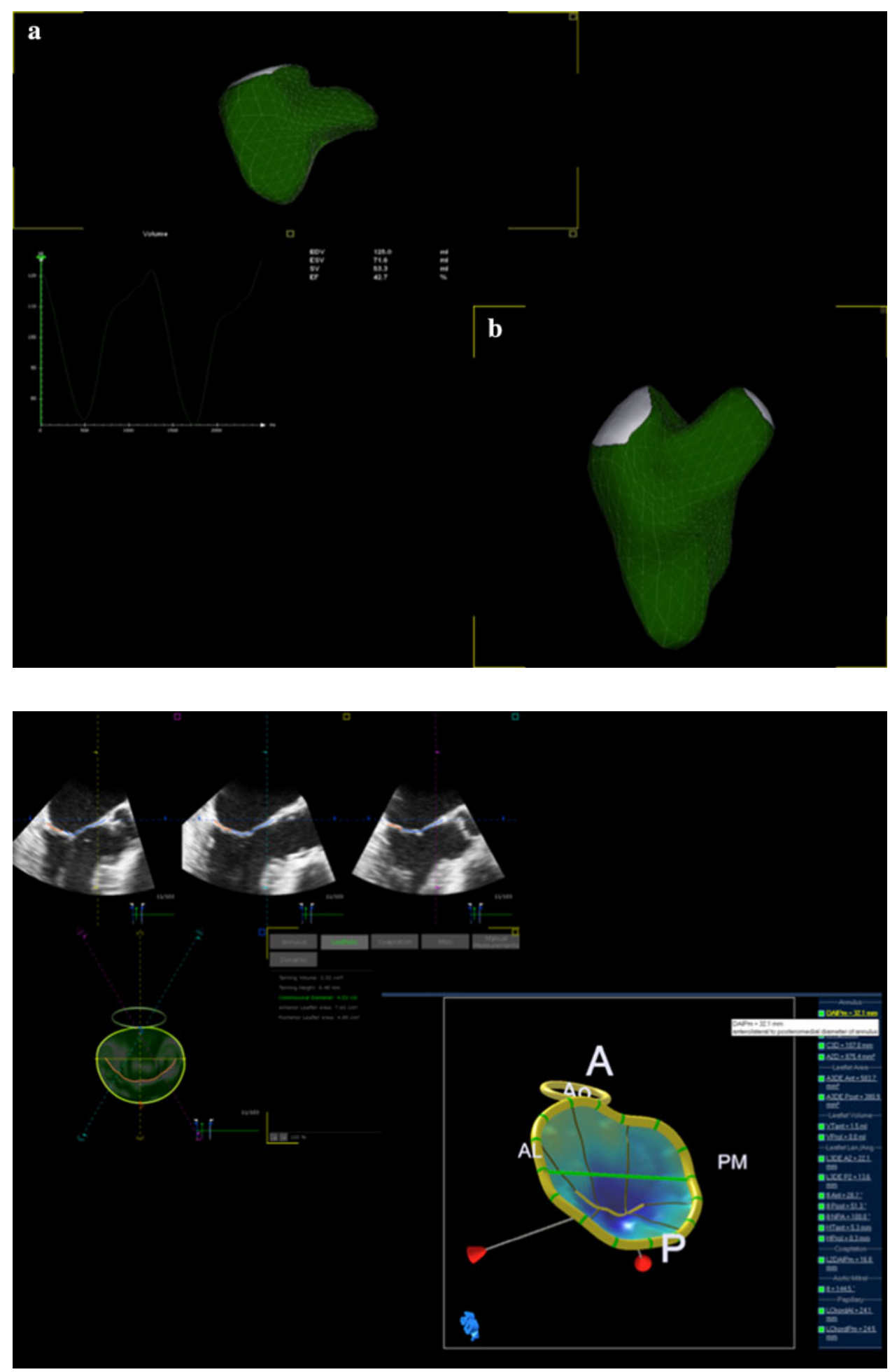

gold standard, especially assessing the posterior leaflet [32]. In addition, 3D echocardiography provides more accurate and less variable measurement of the mitral valve area (MVA) in mitral stenosis compared to standard 2D-based measurements and shows the best agreement with invasive methods [33, 34]. Further, 3D echocardiography has been used for guidance during percutaneous mitral valvuloplasty and has been shown to be a suitable technique for monitoring its efficacy and complications $[35,36]$. In poor surgical candidates presenting with paravalvular leak, 3Dguided placement of the occluding device has proven to be feasible and superior to 2D [37, 38]. Assuming high-quality $3 \mathrm{D}$ datasets, the echocardiographer should not only be able to guide the procedure but also provide accurate 
quantitative information about the paravalvular leak to assist in sizing of the occluding device (Fig. 8).

\section{Aortic Valve}

Compared with the large body of literature featuring the MV, studies assessing the native AV using 3D echocardiography are limited. The AV can be optimally visualized only in $18 \%$ when using RT-3D TEE, most likely because the $\mathrm{AV}$ is an anterior structure with a longer distance from the transducer and with thinner leaflets compared to the MV [39]. However, 3D TEE provides a better understanding of the relationship of the AV and the surrounding structures, such as LVOT, and anterior leaflets of the MV without the need for time-consuming mental reconstruction needed with $2 \mathrm{D}$.

In recent years, the importance of 3D imaging during AV procedures has increased considerably due to the introduction of TAVI. The technique consists of positioning and deployment of a stented bioprosthetic valve over a balloon catheter in the native AV position approached through the retrograde transfemoral or the antegrade transapical access [40-42]. The prosthetic valve is deployed after a balloon valvuloplasty under fluoroscopic guidance. Intraoperative TEE plays an important role to provide (1) accurate measurements of the native AV annular and LVOT for appropriate prosthetic valve sizing, (2) guidance during prosthetic valve positioning, and (3) assessment of the prothetic valve function and the severity of paravalvular leaks after prosthetic valve deployment [43, 44]. Additionally, 3D TEE is helpful in visualizing the degree and distribution of calcifications on and adjacent to the AV and in measuring the distance from the AV leaflets to the left main coronary ostium, the angle of the LVOT to the aorta, and the baseline severity of LV size, function, and hypertrophy.

By utilizing the built-in software, the echocardiographer is able to make accurate measurements of the LVOT and the annulus of the AV. Following the acquisition of a full volume dataset, the image should be aligned optimally using the multiplanar reconstruction (MPR) mode. The coronal "green box" on the MPR mode should display the long axis of the AV. Further adjustments with the "red plane" should be made in order to have the "red box" or saggital plane displaying either the LVOT or the annulus of the AV. Adjustment of the MPR planes is facilitated while playing the cine loop. Great care needs to be taken to avoid inclusion of the anterior leaflet of the MV when assessing the LVOT. After correct identification of the structures to be analyzed, the dimensions and the area of the LVOT and aortic annulus can then be calculated (Fig. 9). Recent guidelines recommend the incorporation of 3D TEE not only for the assessment of the AV but also for guidance of

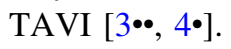

\section{Left Atrial Appendage}

TEE is considered the gold standard imaging modality for detection of LAA thrombi. Despite a relatively high specificity, 2D TEE imaging may overestimate the incidence of thrombi partly due to the complex geometry of multi-lobed LAA. RT-3D TTE or reconstruction 3D TEE
Fig. 8 Three-dimensional TEE color full volume of the paravalvular leak of the MV displayed in three multiplanar reconstruction planes (green, red, and blue MPRs). By utilizing built-in software, the measurement of the diameter and area of the paravalvular leak can be obtained (Color figure online)

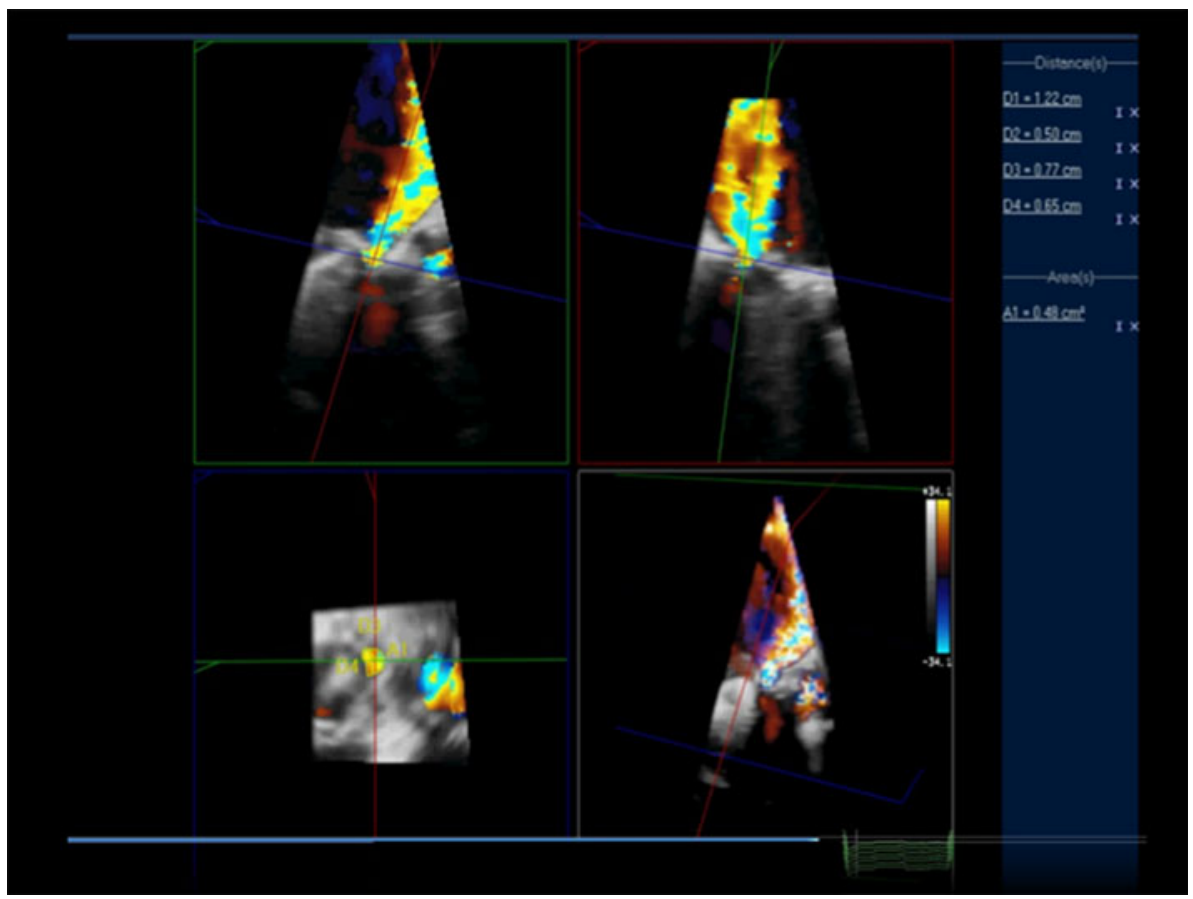


Fig. 9 3D TEE of the AV displayed in the three multiplanar reconstructions (green, red, and blue MPRs) along with the measurements of area and diameters of the aortic valve (bottom) (Color figure online)

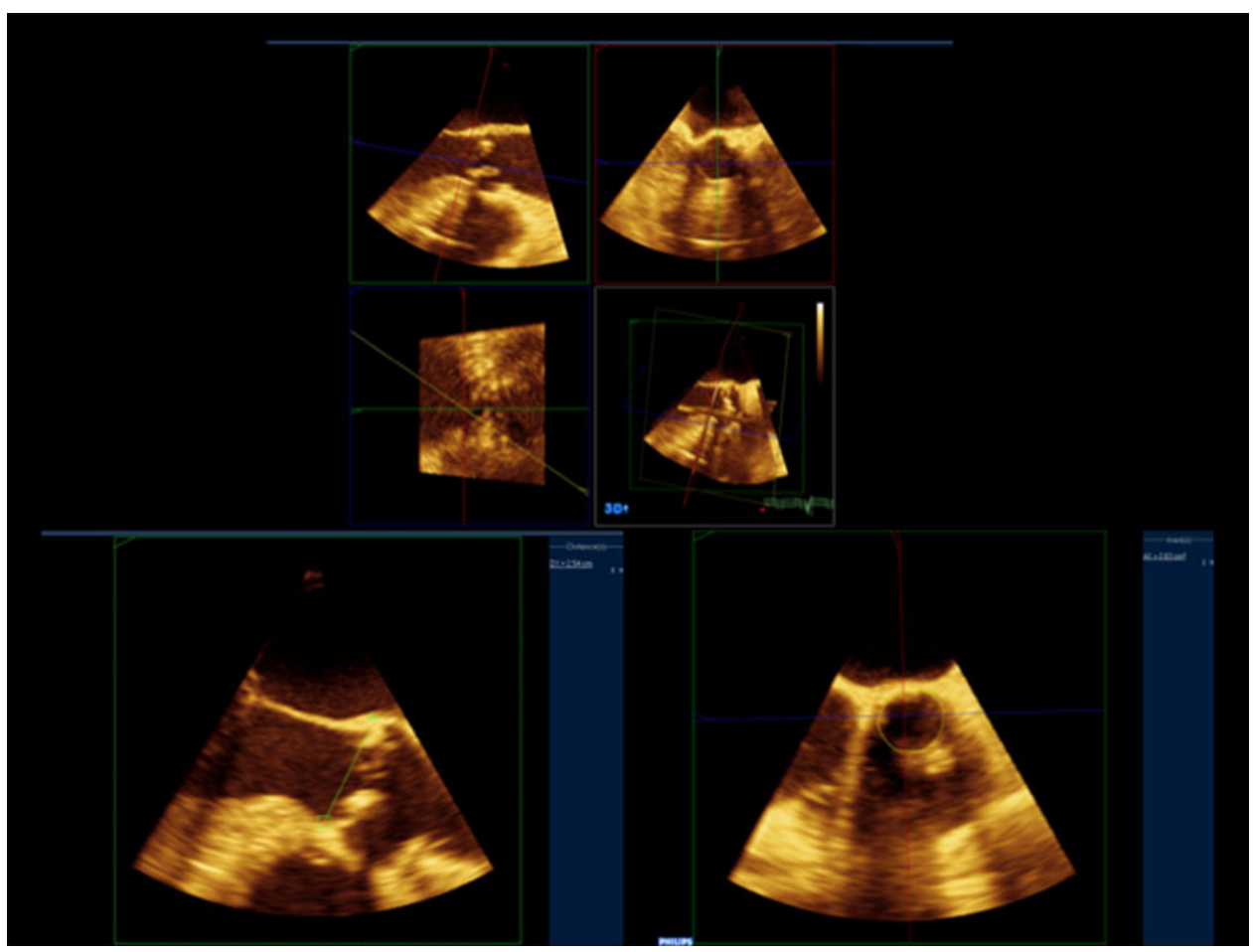

enables excellent visualization of the LAA anatomy and function, and RT-3D TEE provides a higher specificity in defining LAA pathology than 2D TEE [45-47]. Clinically, 3D echocardiography helps to distinguish thrombi from anatomical variants and might alter the course of therapy for patients with atrial fibrillation and help determine the need for additional surgical interventions such as ligation of the LAA in patients undergoing assisted devices placement. The best way to visualize the LAA is by using the focused sector function (zoom) with the adjacent ligament of Marshall. 3D echocardiography is also helpful for guiding percutaneous interventions involving the atrial septum, such as the Lariat procedure [48, 49]. During this intervention, TEE is used as guidance for positioning a marker balloon at the ostium of the LAA and to confirm acute closure of the LAA [50]. During atrial septum defect (ASD) or PFO closures, 3D TEE provided excellent anatomic detail of the septal defects and continuous visualization of the 3D relationships involved in sheath positioning and device deployment [51].

\section{Tricuspid and Pulmonic Valves}

The TV is composed of three leaflets attached to a fibrous annulus presenting a complex three-dimensional shape and thus it is not possible to visualize it in one cross-sectional view using $2 \mathrm{D}$ echocardiography. In this context, 3D echocardiography allows users to visualize the whole TV apparatus leading to better understanding of the pathophysiological mechanisms of underlying TV diseases.
While 3D TTE in patients with good-quality 2D images generated optimal 3D images of the TV in $90 \%$ of patients, 3D TEE of TV can be optimally visualized with RT-3D TEE in only $11 \%$ of cases mainly because of its thin leaflets and anterior position [39, 52]. 3D TTE provides incremental diagnostic value over 2D echocardiography with regard to the etiology and location of the abnormal leaflet segments and the exact shape and size of the vena contracta (VC) [53]. The en face view of the TV may assist in the diagnosis of TV variants such as the presence of a bileaflet TV which has only been described using 3D-TTE [54]. 3D echocardiography can be helpful in identifying whether pacemaker or intracardiac defibrillator leads contribute to tricuspid regurgitation [55]. Further, 3D color flow Doppler showed that the TV VC is more of an elliptic shape compared to the MV [56].

In general, the morphology of the TV is best assessed using the $3 \mathrm{D}$ zoom mode. The 3D full volume mode might add information about the relationship to adjacent structures and help to examine the papillary muscles. Finally, the $3 \mathrm{D}$ color full volume is required for the determination of the VC. However, the insufficient visualization of the TV using RT-3D TEE, which may be additionally compromised by the presence of a pulmonary catheter in patients undergoing cardiac surgery, presents a major limitation of RT-3D TEE for assessment of the TV.

Normal pulmonic valves (PV) are barely visualized by 2D TEE, and thus good 3D TEE images are extremely rare because of their anterior position and thin cusps. Intraoperatively, 3D TTE can perhaps provide better scanning of 
the PV because it offers the capability to change lateral position and lateral width of the image (Fig. 10; Movie clip 6). 3D TEE can be helpful by identifying the location pathology, mechanism of PV dysfunction, and accurate RV outflow tract measurements in patients with congenital RV outflow tract obstruction. Furthermore, 3D TTE improved the accuracy in the assessment of pulmonary regurgitation, leadingto improved timing of surgical intervention [57]. 3D TEE revealed that right ventricular outflow (RVOT) geometry was not generally circular but oval with two different types: horizontal and vertical RVOT shapes. Because of the detailed morphological information of RVOT, 3D TEE could provide more accurate assessments of cardiac output than 2D [58]. More recently, PV replacement using the Medtronic Melody ${ }^{\circledR}$ Transcatheter Pulmonary Valve has been approved by the FDS. Better understanding of RVOT morphology may assist in optimal sizing and better guidance for percutaneous PV implantation.

\section{Assessment of the Ascending Aorta}

According to the ACC/AHA/ASE, the use of TEE for cardiac surgery utilizing cardiopulmonary bypass (CPB) presents a class IIa indication for the assessment of aortic atheroma [59]. However, large sections of the ascending aorta are not visible with 2D TEE due to the interposition of the right mainstem bronchus between the ascending aorta and the esophagus. Epiaortic scanning is considered the gold standard and has been shown to be superior to TEE and surgical palpation in assessing the extent of plaques in the ascending aorta, and might reduce the incidence for neurologic dysfunction [60-62]. A case series using RT-3D epiaortic echocardiography showed that 3D was better in displaying diffusely dispersed plaques. Another advantage of 3D epiaortic scans might be the inclusion of discernable landmarks within the aorta like the AV to clarify the relative position of the plaque [63].

\section{Assessment of Congenital Heart Disease}

3D echocardiography provides incremental information on patients with a variety of complex congenital heart disease. RT-3D TTE and TEE are currently available for visualization of abnormal morphology, assessment of chamber size and ventricular function, and image-guided interventions in the congenital heart disease population [51, 6467]. RT-3D TTE provided superior detail of the mural leaflet and anterior commissural abnormalities for the left atrioventricular valve compared with $2 \mathrm{D}$ echocardiography. 3D TEE has been reported to be useful in the perioperative setting to detect residual defects after closure of ASD or for guiding percutaneous interventions involving the atrial septum [65]. However, 3D TEE imaging is limited to adolescent or adult patients because the 3D TEE probe is essentially equivalent in size to the existing $2 \mathrm{D}$ TEE adult probe.
Fig. $102 D$ and $3 D$ images of a pulmonic bioprosthetic valve. a ME asc aortic SAX view.

Notice the partial opening of the leaflet of the bioprosthetic PV in 2D. b RT-3D TEE of the PV. By changing the $3 \mathrm{D}$ elevation position and width, it was possible to confirm the normal function of the bioprosthetic valve

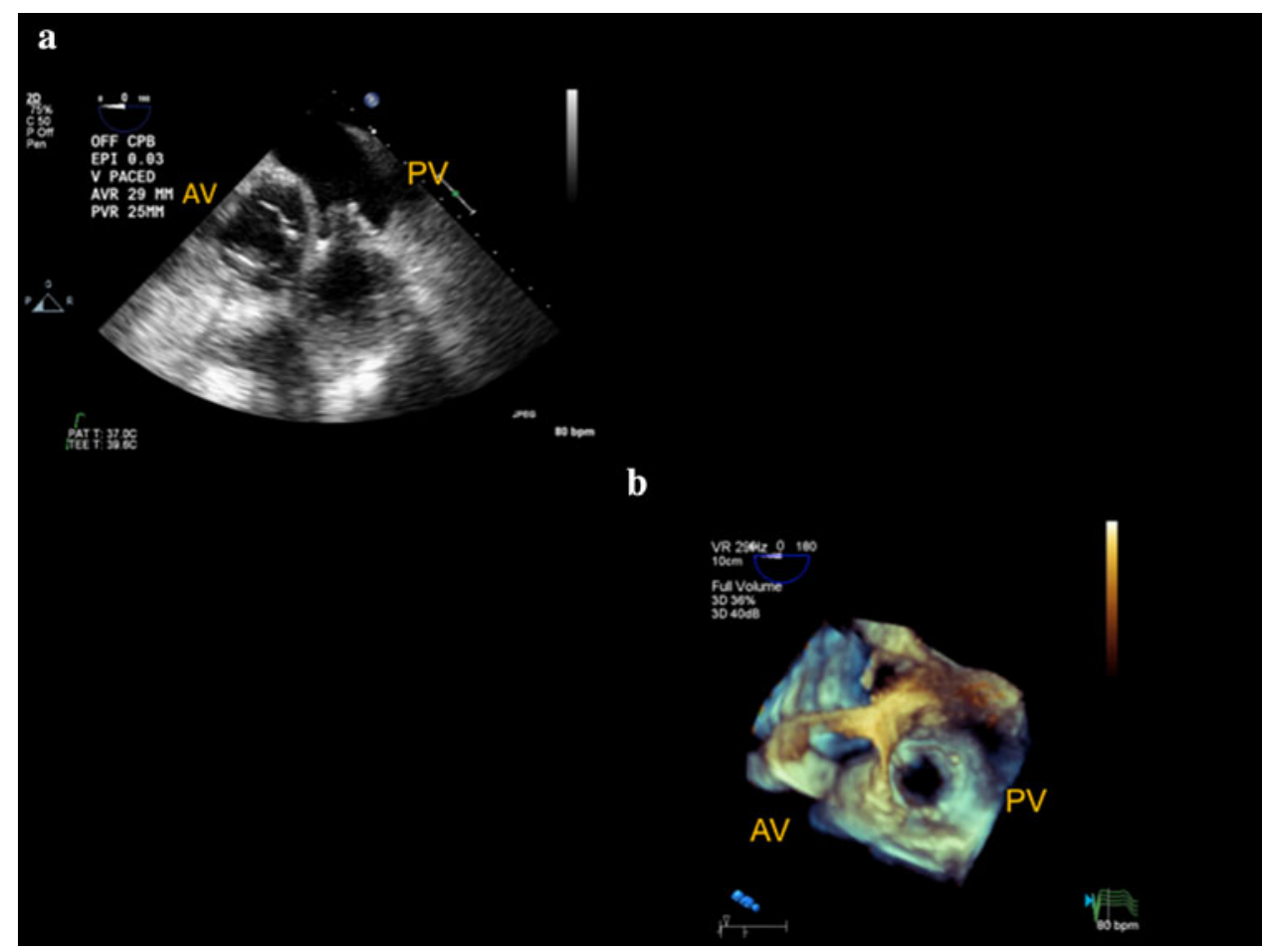




\section{Conclusion and Future Directions}

In conclusion, 3D echocardiography represents a novel clinical and intuitively educational perioperative cardiovascular imaging modality. In addition, live 3D TEE significantly improves the communication of cardiac diagnosis between cardiac surgeons or interventionalists and the intraoperative echocardiographer. However, according to its main critics, the utility of intraoperative 3D TEE is limited to the acquisition of "pretty pictures" rather than its unquestionable impact on surgical decision-making and clinical outcomes. With further technological advances in 3D imaging quality, acquisition speed, and more automated quantification packages, 3D echocardiography is not only here to stay but will become a standard component of perioperative imaging of the patient with cardiac disease.

Ultimately, this technology together with the development of computational capacity and 3D printing tools should open up the door for the creation of precision prosthetics and the establishment of virtual surgery platforms. Although intraoperative 3D TEE is not yet a mature technology, we encourage even the skeptics to embrace it and, more importantly, support its transition towards a standard imaging modality in the perioperative arena.

Disclosures Renata G. Ferreira, Yong Seon Choi, and G. Burkhard Mackensen declare that they have no conflict of interest.

Human and Animal Rights and Informed Consent This article does not contain any studies with human or animal subjects performed by any of the authors.

\section{References}

Papers of particular interest, published recently, have been highlighted as:

- Of importance

•. Of major importance

1. Mor-Avi V, Sugeng L, Lang RM. Real-time 3-dimensional echocardiography: an integral component of the routine echocardiographic examination in adult patients? Circulation. 2009;119:314-29.

2. Hung J, Lang R, Flachskampf F, et al. 3D echocardiography: a review of the current status and future directions. J Am Soc Echocardiogr. 2007;20:213-33.

3. •- Lang RM, Badano LP, Tsang W, et al. EAE/ASE recommendations for image acquisition and display using threedimensional echocardiography. J Am Soc Echocardiogr. 2012;25:3-46. This article reviews the history of $3 D$ echocardiography. It describes the importance and limitations to the $3 D$ echocardiography modes as well as technical aspects of image acquisition.

4. - Smith LA, Dworakowski R, Bhan A, et al. Real-time threedimensional transesophageal echocardiography adds value to transcatheter aortic valve implantation. J Am Soc Echocardiogr.
2013;26:359-69. The practice of replacing AV by transcatheter aortic valve implantation has become more frequent in the recent years. In the majority of places in the USA, TAVI are performed under the guidance of TEE. This article is an important guide for echocardiographers involved in the procedure.

5. Soliman OI, Krenning BJ, Geleijnse ML, et al. Quantification of left ventricular volumes and function in patients with cardiomyopathies by real-time three-dimensional echocardiography: a head-to-head comparison between two different semiautomated endocardial border detection algorithms. J Am Soc Echocardiogr. 2007;20:1042-9.

6. Mor-Avi V, Jenkins C, Kuhl HP, et al. Real-time 3-dimensional echocardiographic quantification of left ventricular volumes: multicenter study for validation with magnetic resonance imaging and investigation of sources of error. JACC Cardiovasc Imaging. 2008;1:413-23.

7. Jenkins C, Bricknell K, Hanekom L, Marwick TH. Reproducibility and accuracy of echocardiographic measurements of left ventricular parameters using real-time three-dimensional echocardiography. J Am Coll Cardiol. 2004;44:878-86.

8. Jacobs LD, Salgo IS, Goonewardena S, et al. Rapid online quantification of left ventricular volume from real-time threedimensional echocardiographic data. Eur Heart J. 2006;27:460-8.

9. Sugeng L, Mor-Avi V, Weinert L, et al. Quantitative assessment of left ventricular size and function: side-by-side comparison of real-time three-dimensional echocardiography and computed tomography with magnetic resonance reference. Circulation. 2006;114:654-61.

10. Corsi C, Lang RM, Veronesi F, et al. Volumetric quantification of global and regional left ventricular function from real-time threedimensional echocardiographic images. Circulation. 2005;112: 1161-70.

11. Bicudo LS, Tsutsui JM, Shiozaki A, et al. Value of real time threedimensional echocardiography in patients with hypertrophic cardiomyopathy: comparison with two-dimensional echocardiography and magnetic resonance imaging. Echocardiography. 2008;25: 717-26.

12. Gutierrez-Chico JL, Zamorano JL, Perez de Isla L, et al. Comparison of left ventricular volumes and ejection fractions measured by threedimensional echocardiography versus by two-dimensional echocardiography and cardiac magnetic resonance in patients with various cardiomyopathies. Am J Cardiol. 2005;95:809-13.

13. Nikitin NP, Constantin $\mathrm{C}$, Loh $\mathrm{PH}$, et al. New generation 3-dimensional echocardiography for left ventricular volumetric and functional measurements: comparison with cardiac magnetic resonance. Eur J Echocardiogr. 2006;7:365-72.

14. Pouleur AC, le Polain de Waroux JB, Pasquet A, et al. Assessment of left ventricular mass and volumes by three-dimensional echocardiography in patients with or without wall motion abnormalities: comparison against cine magnetic resonance imaging. Heart. 2008;94:1050-7.

15. Dorosz JL, Lezotte DC, Weitzenkamp DA, Allen LA, Salcedo EE. Performance of 3-dimensional echocardiography in measuring left ventricular volumes and ejection fraction: a systematic review and meta-analysis. J Am Coll Cardiol. 2012;59:1799-808.

16. Lang RM, Bierig M, Devereux RB, et al. Recommendations for chamber quantification: a report from the American Society of Echocardiography's Guidelines and Standards Committee and the Chamber Quantification Writing Group, developed in conjunction with the European Association of Echocardiography, a branch of the European Society of Cardiology. J Am Soc Echocardiogr. 2005; 18:1440-63.

17. Caiani EG, Corsi C, Sugeng L, et al. Improved quantification of left ventricular mass based on endocardial and epicardial surface detection with real time three dimensional echocardiography. Heart. 2006;92:213-9. 
18. Mor-Avi V, Sugeng L, Weinert L, et al. Fast measurement of left ventricular mass with real-time three-dimensional echocardiography: comparison with magnetic resonance imaging. Circulation. 2004;110:1814-8.

19. Qin JX, Jones M, Travaglini A, et al. The accuracy of left ventricular mass determined by real-time three-dimensional echocardiography in chronic animal and clinical studies: a comparison with postmortem examination and magnetic resonance imaging. J Am Soc Echocardiogr. 2005;18:1037-43.

20. Oe H, Hozumi T, Arai K, et al. Comparison of accurate measurement of left ventricular mass in patients with hypertrophied hearts by real-time three-dimensional echocardiography versus magnetic resonance imaging. Am J Cardiol. 2005;95:1263-7.

21. Miller CA, Pearce K, Jordan P, et al. Comparison of real-time three-dimensional echocardiography with cardiovascular magnetic resonance for left ventricular volumetric assessment in unselected patients. Eur Heart J Cardiovasc Imaging. 2012;13: 187-95.

22. Karhausen J, Dudaryk R, Phillips-Bute B, et al. Three-dimensional transesophageal echocardiography for perioperative right ventricular assessment. Ann Thorac Surg. 2012;94:468-74.

23. Tamborini G, Brusoni D, Torres Molina JE, et al. Feasibility of a new generation three-dimensional echocardiography for right ventricular volumetric and functional measurements. Am J Cardiol. 2008;102:499-505.

24. Ben Zekry S, Nagueh SF, Little SH, et al. Comparative accuracy of two- and three-dimensional transthoracic and transesophageal echocardiography in identifying mitral valve pathology in patients undergoing mitral valve repair: initial observations. J Am Soc Echocardiogr. 2011;24:1079-85.

25. Biaggi P, Jedrzkiewicz S, Gruner C, et al. Quantification of mitral valve anatomy by three-dimensional transesophageal echocardiography in mitral valve prolapse predicts surgical anatomy and the complexity of mitral valve repair. J Am Soc Echocardiogr. 2012;25:758-65.

26. Grewal J, Mankad S, Freeman WK, et al. Real-time threedimensional transesophageal echocardiography in the intraoperative assessment of mitral valve disease. J Am Soc Echocardiogr. 2009;22:34-41.

27. Manda J, Kesanolla SK, Hsuing MC, et al. Comparison of real time two-dimensional with live/real time three-dimensional transesophageal echocardiography in the evaluation of mitral valve prolapse and chordae rupture. Echocardiography. 2008;25: 1131-7.

28. Bartels K, Thiele RH, Phillips-Bute B, et al. Dynamic indices of mitral valve function using perioperative three-dimensional transesophageal echocardiography. J Cardiothorac Vasc Anesth. 2013; in press.

29. Little SH, Ben Zekry S, Lawrie GM, Zoghbi WA. Dynamic annular geometry and function in patients with mitral regurgitation: insight from three-dimensional annular tracking. J Am Soc Echocardiogr. 2010;23:872-9.

30. Mahmood F, Warraich HJ, Shahul S, et al. En face view of the mitral valve: definition and acquisition. Anesth Analg. 2012;115: 779-84.

31. Chikwe J, Adams DH, Su KN, et al. Can three-dimensional echocardiography accurately predict complexity of mitral valve repair? Eur J Cardiothorac Surg. 2012;41:518-24.

32. Jungwirth B, Adams DB, Mathew JP, Swaminathan M, Glower DD, Mackensen GB. Mitral valve prolapse and systolic anterior motion illustrated by real time three-dimensional transesophageal echocardiography. Anesth Analg. 2008;107:1822-4.

33. Chu JW, Levine RA, Chua $\mathrm{S}$, et al. Assessing mitral valve area and orifice geometry in calcific mitral stenosis: a new solution by real-time three-dimensional echocardiography. J Am Soc Echocardiogr. 2008;21:1006-9.
34. Zamorano J, Cordeiro P, Sugeng L, et al. Real-time threedimensional echocardiography for rheumatic mitral valve stenosis evaluation: an accurate and novel approach. J Am Coll Cardiol. 2004;43:2091-6.

35. Zamorano J, Perez de Isla L, Sugeng L, et al. Non-invasive assessment of mitral valve area during percutaneous balloon mitral valvuloplasty: role of real-time 3D echocardiography. Eur Heart J. 2004;25:2086-91.

36. Langerveld J, Valocik G, Plokker HW, et al. Additional value of three-dimensional transesophageal echocardiography for patients with mitral valve stenosis undergoing balloon valvuloplasty. J Am Soc Echocardiogr. 2003;16:841-9.

37. Shapira Y, Hirsch R, Kornowski R, et al. Percutaneous closure of perivalvular leaks with Amplatzer occluders: feasibility, safety, and shortterm results. J Heart Valve Dis. 2007;16:305-13.

38. Becerra JM, Almeria C, de Isla LP, Zamorano J. Usefulness of 3D transoesophageal echocardiography for guiding wires and closure devices in mitral perivalvular leaks. Eur J Echocardiogr. 2009;10:979-81.

39. Sugeng L, Shernan SK, Salgo IS, et al. Live 3-dimensional transesophageal echocardiography initial experience using the fully-sampled matrix array probe. J Am Coll Cardiol. 2008;52: 446-9.

40. Walther T, Thielmann M, Kempfert J, et al. PREVAIL TRANSAPICAL: multicentre trial of transcatheter aortic valve implantation using the newly designed bioprosthesis (SAPIENXT) and delivery system (ASCENDRA-II). Eur J Cardiothorac Surg. 2012;42:278-83 discussion 83.

41. Strauch JT, Scherner M, Haldenwang PL, et al. Transapical minimally invasive aortic valve implantation and conventional aortic valve replacement in octogenarians. Thorac Cardiovasc Surg. 2012;60:335-42.

42. van der Boon RM, Nuis RJ, Van Mieghem NM, et al. Clinical outcome following transcatheter aortic valve implantation in patients with impaired left ventricular systolic function. Catheter Cardiovasc Interv. 2012;79:702-10.

43. Moss RR, Ivens E, Pasupati S, et al. Role of echocardiography in percutaneous aortic valve implantation. JACC Cardiovasc Imaging. 2008;1:15-24.

44. Kennedy C, Lien RA, Stolz E, van Joost T, Naafs B. Leprosy and human immunodeficiency virus infection. A closer look at the lesions. Int J Dermatol. 1990;29:139-40.

45. Mizuguchi KA, Burch TM, Bulwer BE, Fox AA, Rizzo RJ, Shernan SK. Thrombus or bilobar left atrial appendage? Diagnosis by real-time three-dimensional transesophageal echocardiography. Anesth Analg. 2009;108:70-2.

46. Cummisford K, Sundar S, Hagberg R, Mahmood F. Real-time three-dimensional transesophageal echocardiography and a congenital bilobar left atrial appendage. J Cardiothorac Vasc Anesth. 2010;24:475-7.

47. Agoston I, Xie T, Tiller FL, Rahman AM, Ahmad M. Assessment of left atrial appendage by live three-dimensional echocardiography: early experience and comparison with transesophageal echocardiography. Echocardiography. 2006;23:127-32.

48. Massumi A, Chelu MG, Nazeri A, et al. Initial experience with a novel percutaneous left atrial appendage exclusion device in patients with atrial fibrillation, increased stroke risk, and contraindications to anticoagulation. Am J Cardiol. 2013;111: 869-73.

49. Bartus K, Han FT, Bednarek J, et al. Percutaneous left atrial appendage suture ligation using the LARIAT device in patients with atrial fibrillation: initial clinical experience. J Am Coll Cardiol. 2012;. doi:10.1016/j.jacc.2012.06.046.

50. Bartus K, Bednarek J, Myc J, et al. Feasibility of closed-chest ligation of the left atrial appendage in humans. Heart Rhythm. 2011;8:188-93. 
51. Baker GH, Shirali G, Ringewald JM, Hsia TY, Bandisode V. Usefulness of live three-dimensional transesophageal echocardiography in a congenital heart disease center. Am J Cardiol. 2009;103:1025-8.

52. Badano LP, Agricola E, Perez de Isla L, Gianfagna P, Zamorano JL. Evaluation of the tricuspid valve morphology and function by transthoracic real-time three-dimensional echocardiography. Eur J Echocardiogr. 2009;10:477-84.

53. Pothineni KR, Duncan K, Yelamanchili P, et al. Live/real time three-dimensional transthoracic echocardiographic assessment of tricuspid valve pathology: incremental value over the twodimensional technique. Echocardiography. 2007;24:541-52.

54. Anwar AM, Attia WM, Nosir YF, El-Amin AM. Unusual bileaflet tricuspid valve by real time three-dimensional echocardiography. Echocardiography. 2008;25:534-6.

55. Nucifora G, Badano LP, Allocca G, et al. Severe tricuspid regurgitation due to entrapment of the anterior leaflet of the valve by a permanent pacemaker lead: role of real time three-dimensional echocardiography. Echocardiography. 2007;24:649-52.

56. Sugeng L, Weinert L, Lang RM. Real-time 3-dimensional color Doppler flow of mitral and tricuspid regurgitation: feasibility and initial quantitative comparison with 2-dimensional methods. J Am Soc Echocardiogr. 2007;20:1050-7.

57. Pothineni KR, Wells BJ, Hsiung MC, et al. Live/real time threedimensional transthoracic echocardiographic assessment of pulmonary regurgitation. Echocardiography. 2008;25:911-7.

58. Izumo M, Shiota M, Saitoh T, et al. Non-circular shape of right ventricular outflow tract: a real-time 3-dimensional transesophageal echocardiography study. Circ Cardiovasc Imaging. 2012;5: 621-7.

59. Cheitlin MD, Armstrong WF, Aurigemma GP, et al. ACC/AHA/ ASE 2003 guideline update for the clinical application of echocardiography: summary article: a report of the American college of cardiology/American heart association task force on practice guidelines (ACC/AHA/ASE committee to update the 1997 guidelines for the clinical application of echocardiography). Circulation. 2003;108:1146-62.

60. Wilson MJ, Boyd SY, Lisagor PG, Rubal BJ, Cohen DJ. Ascending aortic atheroma assessed intraoperatively by epiaortic and transesophageal echocardiography. Ann Thorac Surg. 2000; 70:25-30.

61. Rosenberger P, Shernan SK, Loffler M, et al. The influence of epiaortic ultrasonography on intraoperative surgical management in 6051 cardiac surgical patients. Ann Thorac Surg. 2008;85: 548-53.

62. Glas KE, Swaminathan M, Reeves ST, et al. Guidelines for the performance of a comprehensive intraoperative epiaortic ultrasonographic examination: recommendations of the American society of echocardiography and the society of cardiovascular anesthesiologists; endorsed by the society of thoracic surgeons. Anesth Analg. 2008;106:1376-84.

63. Bainbridge DT, Murkin JM, Menkis A, Kiaii B. The use of 3D epiaortic scanning to enhance evaluation of atherosclerotic plaque in the ascending aorta: a case series. Heart Surg Forum. 2004;7:E636-8.

64. De Castro S, Caselli S, Papetti F, et al. Feasibility and clinical impact of live three-dimensional echocardiography in the management of congenital heart disease. Echocardiography. 2006;23:553-61.

65. Shirali GS. Three-dimensional echocardiography in congenital heart disease. Echocardiography. 2012;29:242-8.

66. Takahashi K, Mackie AS, Rebeyka IM, et al. Two-dimensional versus transthoracic real-time three-dimensional echocardiography in the evaluation of the mechanisms and sites of atrioventricular valve regurgitation in a congenital heart disease population. J Am Soc Echocardiogr. 2010;23:726-34.

67. Simpson JM, Miller O. Three-dimensional echocardiography in congenital heart disease. Arch Cardiovasc Dis. 2011;104:45-56. 